\title{
Event-driven mathematical framework for noisy integrate-and-fire neuron networks: spike trains statistics via stochastic calculus, network analysis inspired by queuing theory and an event-driven simulator
} Jonathan Touboul*, Olivier Faugeras and Olivier Rochel

Address: Odyssee Laboratory, INRIA/ENPC/ENS, INRIA Sophia Antipolis, France

Email: Jonathan Touboul* - Jonathan.Touboul@sophia.inria.fr

* Corresponding author

from Sixteenth Annual Computational Neuroscience Meeting: CNS*2007

Toronto, Canada. 7-12 July 2007

Published: 6 July 2007

BMC Neuroscience 2007, 8(Suppl 2):P29 doi:10.1 I86/I47|-2202-8-S2-P29

(C) 2007 Touboul et al; licensee BioMed Central Ltd.

\section{Motivation}

The dynamics of neural networks in the brain is greatly influenced by noise. In the nervous system, sources of noise are everywhere (in the stimulus, in the uncorrelated activity, in the synapses, in the channels), and the emergent phenomena related to these random events such as spontaneous spiking and random collective behaviours are of special importance in the study of the neural code.

Usually, networks of integrate-and-fire neurons with a noisy external drive are studied using the Fokker-Planck equation [1]. Under the assumption of sparse random connectivity, it has been shown that the network dynamics can be in one of two regimes, depending on the parameters: a desynchronized stationary regime, and a weakly synchronized oscillatory regime. However, this approach does not seem to be fully satisfactory because it cannot be easily applied to more general neuron models.

\section{Mathematical approach: bridging neuroscience and communication networks theory}

We propose a framework inspired by the communication network theory to study this type of networks. In contrast with classical analysis (considering the membrane potential of the cells), we consider an event-based description of the network and define a Markov process related to the times of the spikes, the countdown process.
We show that this biologically inspired model is formally equivalent to a class of stochastic networks studied in the field of probability theory [2,3]. Our work consists in generalizing the results obtained in this field to the more intricate biologically-inspired model, and address new questions of special interest for the biological applications under this framework.

\section{Spike train statistics}

In this model, the probability law of the time of the first spike is a fundamental parameter, hence we need to characterize the spike trains statistics as fast and accurately as possible. To this aim, we review and extend some methods coming from stochastic analysis. For instance we show that for classical integrate-and-fire models, the problem reduces to finding the hitting time of a curve by the Brownian motion, using the Dubins-Schwarz' theorem of local martingales representation.

\section{Event-driven stochastic simulator}

This approach leads to a natural event-driven simulation strategy we implemented by extending the software Mvaspike[4]. We show some simulation results illustrating transient behaviours of the network.

\section{Acknowledgements}

The authors warmly acknowledge Denis Talay for fruitful discussions on stochastic analysis and Romain Brette for the network analysis. This work 
was supported by the European Commission (FACETS Project, FP6-2004ISTFET).

\section{References}

I. Brunel N, Hakim V: Fast global oscillations in networks of integrate-and-fire neurons with low firing rates. Neural Comput 1999, I I:|62|-167|.

2. Cottrell M: Mathematical analysis of a neural network with inhibitory coupling. Stochastic Processes and their Applications 1992, 40:103-127.

3. Fricker C, Robert P, Saada E, Tibi D: Analysis of some networks with interaction. Annals of Applied Probability 1994, 4: I I 12 - I I 28.

4. Rochel O, Martinez D: An event-driven framework for the simulation of networks of spiking neurons. Proc European Symposium On Artificial Neural Networks - ESANN 2003:295-300.

Publish with Bio Med Central and every scientist can read your work free of charge

"BioMed Central will be the most significant development for disseminating the results of biomedical research in our lifetime. "

Sir Paul Nurse, Cancer Research UK

Your research papers will be:

- available free of charge to the entire biomedical community

- peer reviewed and published immediately upon acceptance

- cited in PubMed and archived on PubMed Central

- yours - you keep the copyright

Submit your manuscript here:

http://www.biomedcentral.com/info/publishing_adv.asp 